\title{
Longer-Term Baerveldt to Trabectome Glaucoma Surgery Comparison using Propensity Score Matching
}

Tigran Kostanyan, ${ }^{1 \dagger}$ Tarek Shazly, ${ }^{1+}$ Kevin B. Kaplowitz, ${ }^{2}$ Steven Z. Wang, ${ }^{1}$ Sushma Kola, ${ }^{1}$ Eric N. Brown, ${ }^{3}$ Nils A. Loewen ${ }^{1 *}$

1. Department of Ophthalmology, University of Pittsburgh, Pittsburgh, PA

2. Department of Ophthalmology, VA Loma Linda, Loma Linda University, Loma Linda, CA

3. Department of Ophthalmology, Vanderbilt University Medical Center, Nashville, TN

${ }^{\dagger}$ Authors have equally contributed.

*corresponding Author:

Nils A. Loewen, MD, PhD

University of Pittsburgh, School of Medicine

Department of Ophthalmology

203 Lothrop St

Pittsburgh, PA 15213

Email: loewen.nils@gmail.com

Phone: 412-944-2554 Fax: 412-647-5119 


\section{Abstract}

Purpose: To apply propensity score matching to compare Baerveldt glaucoma drainage implants (BGI) to trabectome-mediated ab interno trabeculectomy (AIT). Recent data suggests that AIT can produce results similar to BGI which is traditionally reserved for more severe glaucoma.

Methods: BGI and AIT patients with at least one year of follow-up were included. The primary outcome measures were intraocular pressure (IOP), number of glaucoma medications, and a Glaucoma Index (GI) score. GI reflected glaucoma severity based on visual field, the number of preoperative medications, and preoperative IOP. Score matching used a genetic algorithm consisting of age, gender, type of glaucoma, concurrent phacoemulsification, baseline number of medications, and baseline IOP. Patients with neovascular glaucoma, with prior glaucoma surgery or without a close match were excluded.

Results: Of 353 patients, 30 AIT patients were matched to 29 BGI patients. Baseline characteristics including, IOP, the number of glaucoma medications, type of glaucoma, the degree of VF loss and GI were not significantly different between AIT and BGI. BGI had a preoperative IOP of $21.6 \pm 6.3 \mathrm{mmHg}$ compared to 21.5 \pm 7.4 for AIT on $2.8 \pm 1.1$ medications and $2.5 \pm 2.3$, respectively. At 30 months, the mean IOP was

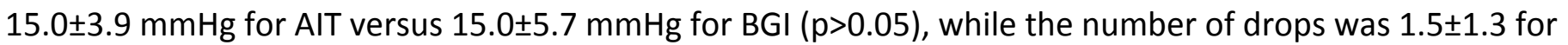
AIT (change: $p=0.001$ ) versus $2.4 \pm 1.2$ for BGI (change: $p=0.17$; AIT vs BGI: 0.007 ). Success, defined as IOP $<21 \mathrm{mmHg},<20 \%$ reduction and no reoperation, was achieved at 1 year in $56 \%$ of AIT versus $55 \%$ of BGI $(p>0.05)$ and $50 \%$ versus $52 \%$ at 2.5 years.

Conclusions: A propensity score matched comparison of AIT and BGI demonstrated a similar IOP reduction through one year. AIT required fewer medications. 


\section{Introduction}

The non-valved Baerveldt glaucoma implant (BGI; Abbott Medical Optics, Abbott Park, IL, USA) was introduced in 1990 [1] and is one of the more commonly used implants in moderate to severe glaucoma [2]. The BGI allows aqueous humor in the anterior chamber to bypass the conventional outflow system into a subconjunctival space maintained by the barium-impregnated, silicone rubber plate of the implant [3]. The $B G I$ is more effective than the valved Ahmed glaucoma implant (AGI) in achieving a lower failure rate and a lower IOP on fewer medications than AGIs at the expense of a higher risk of hypotony [4].

We had recently performed a comparison of AGIs to Trabectome (Neomedix, Tustin, CA, USA) ab interno trabeculectomies (AIT) by applying propensity score matching to achieve equivalent baseline conditions in both [5]. Although AIT is a microincisional glaucoma surgery that uses plasma to molecularize and aspirate the trabecular meshwork thereby enhancing conventional outflow, our study showed a surprisingly similar IOP reduction that was achieved with fewer medications and fewer complications. In another analysis, we stratified AIT outcomes by glaucoma severity and found that a larger IOP and medication decrease can be achieved in more severe glaucoma [6-8]. Other investigators have used AIT after failed glaucoma drainage implants [9] or failed trabeculectomy [10,11] reflecting these observations.

In the present study, we propensity score matched cases of primary BGI and AIT's to directly compare a large and highly effective epibulbar drainage implant to microsurgical trabecular ablation since the traditional indications are too different to allow for prospective randomization.

\section{Methods}

This retrospective analysis was approved by the Institutional Review Board of the University of Pittsburgh (PRO14100026) in accordance with the Declaration of Helsinki and the Health Insurance Portability and Accountability Act. No consent was required due to the retrospective nature of the study. We 
retrospectively reviewed the medical records of patients who underwent BGI or AIT between March of 2012 through August of 2014, were over 18 years old and with or without phacoemulsification. Patients with neovascular glaucoma or prior glaucoma surgery were excluded.

The cohorts were compared using propensity-score case matching using a genetic algorithm based on age, gender, type of glaucoma, concurrent phacoemulsification, and baseline IOP [12,13]. Cases of AIT or BGI too different from their counterparts were excluded. The main outcome measure was the mean IOP reduction. Secondary measures were reduction of medications and failure rates. Failure (using criteria of the Tube versus Trabeculectomy trial) [14] was defined as any one of IOP $<6$, IOP $>21$, or a smaller than $20 \%$ decrease from baseline at any two consecutive visits after the 90 day immediate postoperative period, or the need for a subsequent glaucoma procedure.

\section{Surgical Technique}

Procedures were performed by the same group of surgeons on comparable patient populations for primary and secondary open angle glaucomas and chronic angle closure. AIT was done before phacoemulsification in combined cases. Approximately 140-160 degrees of nasal TM was ablated under direct gonioscopic view. BGI's were inserted through a fornix-based conjunctival flap in the superotemporal quadrant when possible ( $92 \%$ of cases). The tube was positioned on the anterior surface of the iris. All patients received topical fluoroquinolone and prednisolone acetate $1 \%$ four times daily. AIT patients were also given pilocarpine $2 \%$ four times daily for 1 month then twice daily for one month. All preoperative glaucoma medications were stopped on the day of surgery and then added back at the discretion of the surgeon. Postoperative visits at day 1 , week 1 , month 1 , month 3 , month 6 , month 12 , month 18 , month 24 and month 30 were analyzed.

\section{Statistics}

Cases were matched with propensity scores (package "Matching" in R) $[15,16]$ using a genetic 
algorithm based on age, gender, type of glaucoma, concurrent phacoemulsification, baseline IOP and medications. Cases of AIT or BGI too different from their counterparts were excluded. Weighted linear regression was significant when $\mathrm{p}<0.05$. A Kaplan-Meier survival curve for right-censored data with $80 \%$ confidence intervals was computed. A Cox proportional hazard model was developed to estimate the effects of preoperative IOP on surgical success.

\section{Results}

A total of 353 patients met the inclusion criteria. Two hundred and fifty seven eyes were excluded for the occurrence of concurrent surgeries or short follow up. Of the 257 eyes that were excluded, 178 were from the AIT group and 79 were from the BGI group. After propensity score matching 37 eyes from the AIT group and three eyes from the BGI group were excluded. The final cohort included 30 AIT's and 29 BGI's of 59 patients (Fig 1). Using weighted means, there was no significant difference between the two groups for any of the demographic variables measured including age, gender, type of glaucoma, baseline IOP and the number of medications (all $p>0.05$ ) (Table 1). 


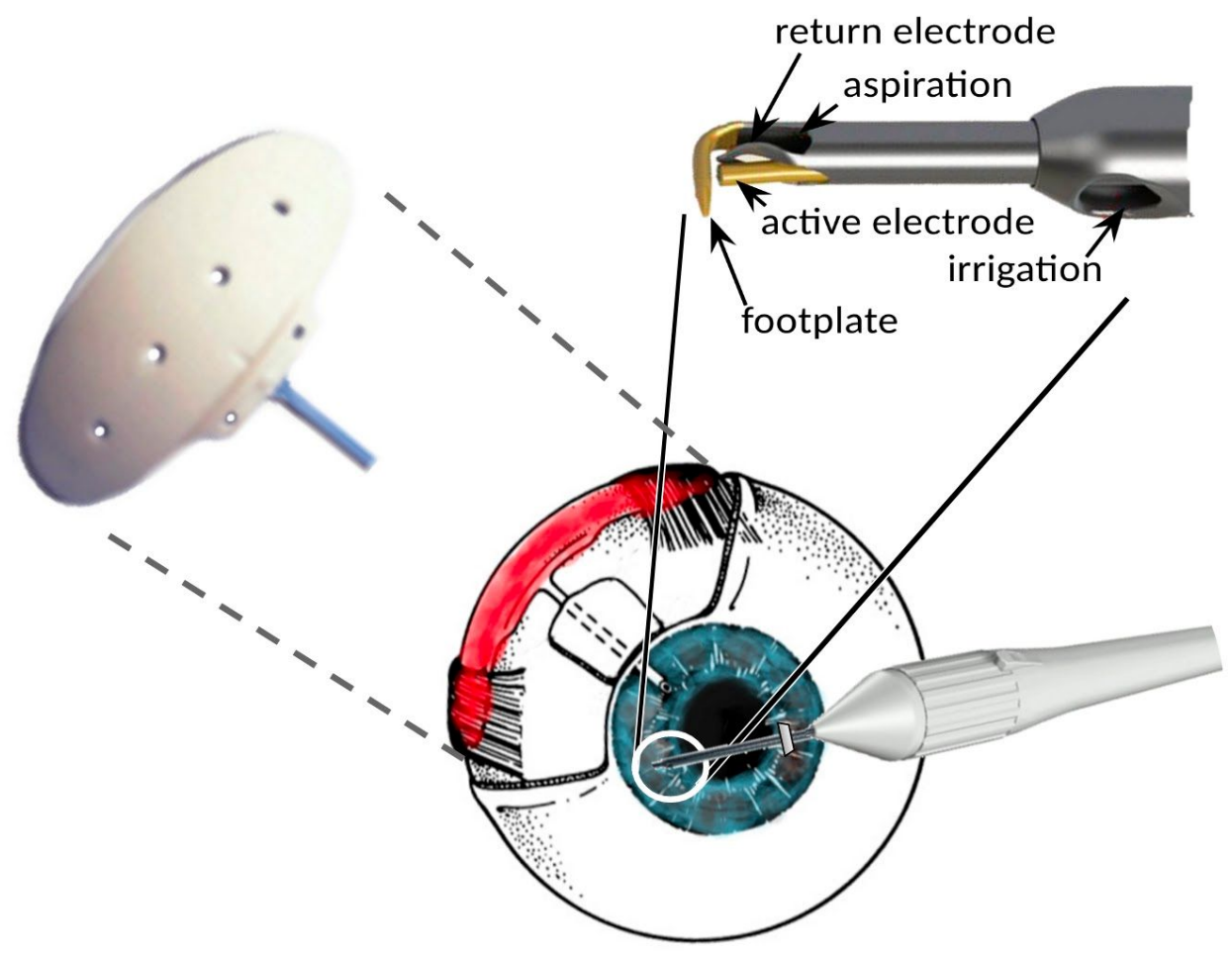

Fig 1. Baerveldt glaucoma drainage implant (BGI) and Trabectome-mediated ab interno trabeculectomy. The BGI (left and center (red)) is most commonly inserted under the superior and lateral recti muscles. AIT is performed along the nasal angle (temporal ablation with nasal incision depicted in this illustration). The tip of the trabectome has a protective footplate that feeds the trabecular meshwork towards the active and passive electrode where plasma is generated (top right). The molecularized tissue is removed by aspiration and irrigation.

Table 1. Weighed Baseline Demographics with ab interno trabeculectomy (AIT) versus Baerveldt Glaucoma Implant (BGI). SD: standard deviation.

$\begin{array}{ccc}\text { BGI } & \text { AIT } & \text { p-value } \\ \text { mean } \pm S D & \text { mean } \pm S D) & \end{array}$

n

29

30

Age, years old (range)

$69.1 \pm 11.6$

$68.3 \pm 12.5$

0.83

Female, \%

46.4

44.6

0.91

Type of glaucoma

0.56 
POAG, \%

CACG, \%

Pigmentary, \%

Other glaucoma, \%

Cases with phacoemulsification, (\%)

Baseline IOP ( $\mathrm{mmHg}$ )

Baseline \# Medications
71.4

76.8

10.7

10.7

7.14

10.7

53.6

$21.6 \pm 6.3$

$2.7 \pm 1.2$
3.57

8.93

53.6

1.00

$21.5 \pm 7.4$

0.95

$2.5 \pm 1.8$

0.57

Both groups started at a statistically similar IOP of $21.6 \pm 6.3$ for BGI and $21.5 \pm 7.4 \mathrm{mmHg}$ for AIT, $\mathrm{p}=0.95$ (Fig 2). After 2.5 years, the IOP for BGI and AIT decreased to $15.0 \pm 5.7 \mathrm{mmHg}$ and $15.0 \pm 3.9 \mathrm{mmHg}$ $(p=0.43)$, respectively.

\section{IOP in BGI and AIT}

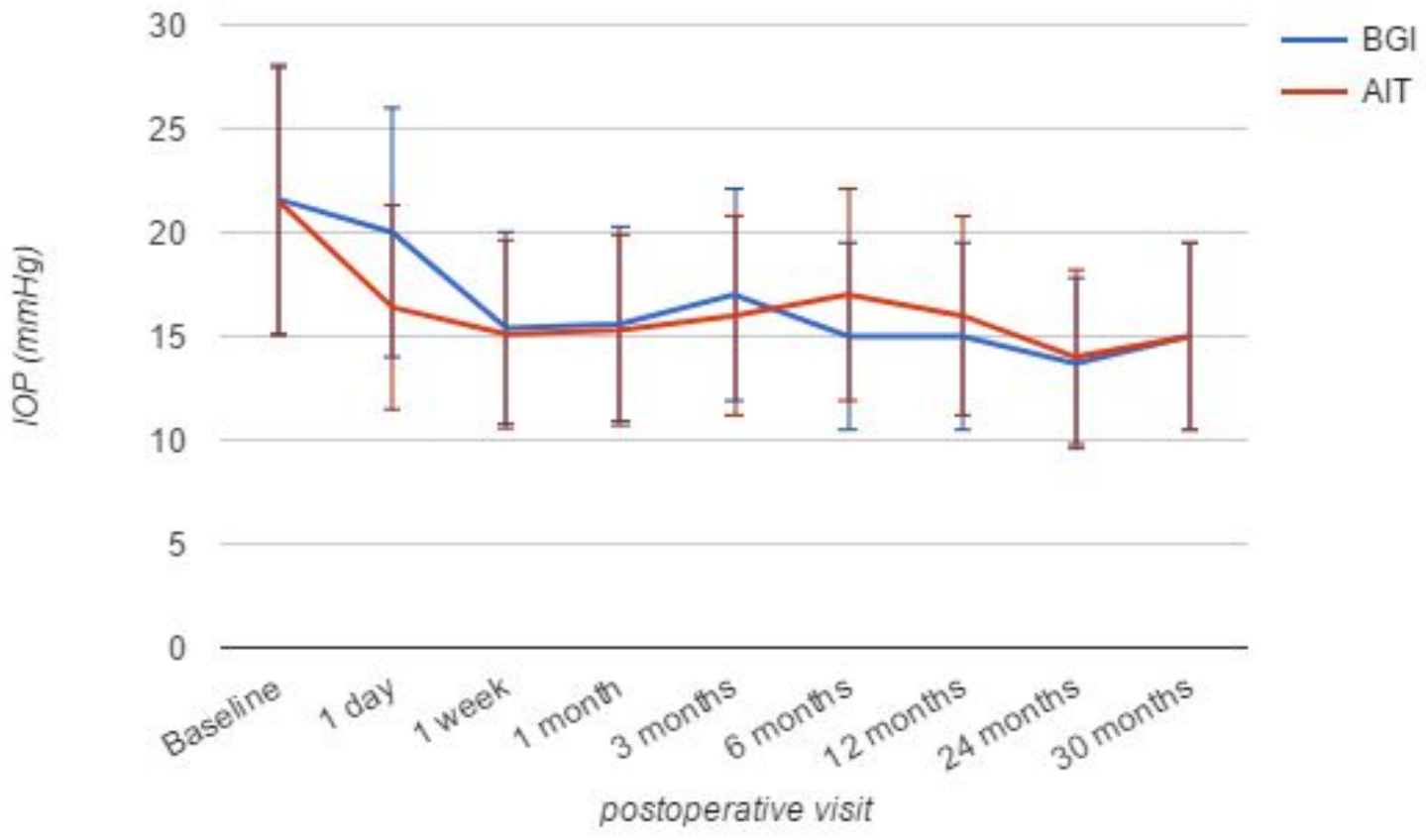

Fig 2. IOP of patients with BGI and AIT. Intraocular pressures (IOP) in Baerveldt glaucoma drainage implants (BGI) and Trabectome ab interno trabeculectomies (AIT) were similar during the time under study. 
As with IOP, there were no statistically significant differences in medication use. The BGI group averaged 2.8 \pm 1.1 medications at baseline and $2.4 \pm 1.2$ at 2.5 years ( $p=0.17$ ) (Fig 3). The AIT group averaged 2.3 \pm 1.2 medications at baseline, which decreased to $1.5 \pm 1.3$ years (change: $p=0.001$; AIT vs BGI: 0.007 ).

\section{Glaucoma Medications in BGI and AIT}

6
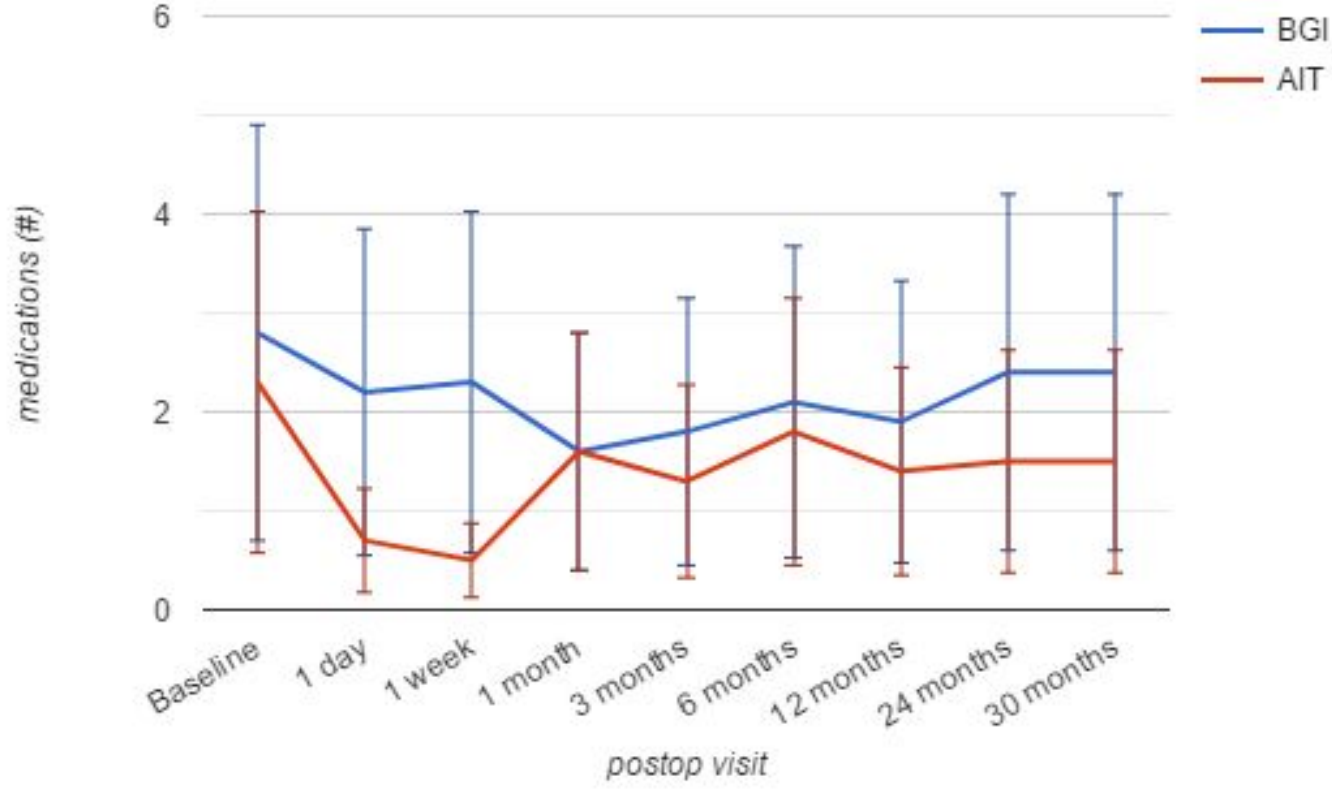

Fig 3. Glaucoma medications in BGI and AIT. in Baerveldt glaucoma drainage implants (BGI) and Trabectome ab interno trabeculectomies (AIT) were similar during the time under study.

Failure was defined as any one of IOP $<6,1 \mathrm{IOP}>21$, or a smaller than $20 \%$ decrease from baseline at any two consecutive visits after the 90 day immediate postoperative period, or the need for a subsequent glaucoma procedure. Success, defined as IOP $<21 \mathrm{mmHg},<20 \%$ reduction and no reoperation, was achieved in $56 \%$ of AIT versus $55 \%$ of BGI at 1 year ( $p>0.05$ ) and $50 \%$ versus $52 \%$ at 2.5 years (Fig 4). 


\section{Kaplan-Meier Survival Curve}

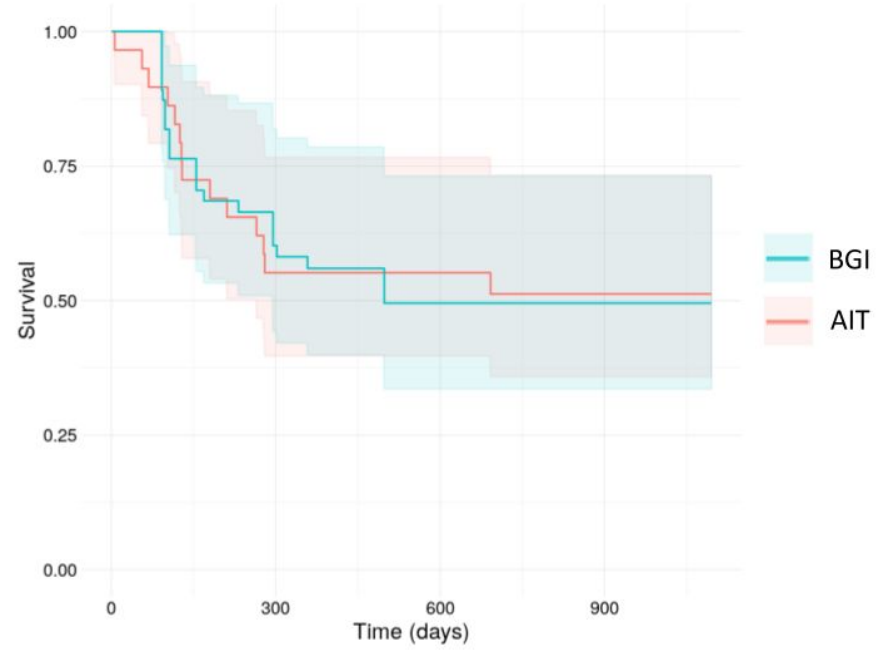

Fig 4. Kaplan Meier Survival of BGI and AIT. Performance of Baerveldt glaucoma drainage implants (BGI) and ab interno trabeculectomy (AIT) was similar in patients matched preoperatively.

When we modeled survival with a Cox hazard model and a preoperative IOP variable of $16 \mathrm{mmHg}$, approximately about 40\% survived at 1 year before stabilizing at 30\% (Fig 5). There was no difference between the two groups. When the IOP variable was increased to $21 \mathrm{mmHg}$, a stable survival of $50 \%$ was achieved at two years both AIT and BGI. With baseline IOP set to $26 \mathrm{mmHg}$, survival was $75 \%$ at 500 days and onward for both groups.

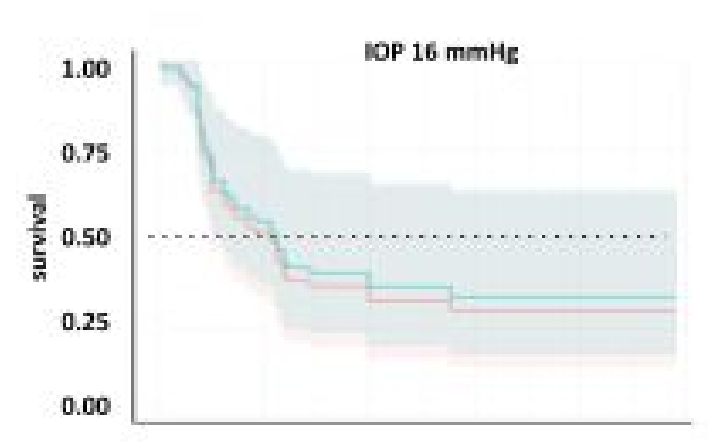

\section{Cox Proportional Hazards Model}
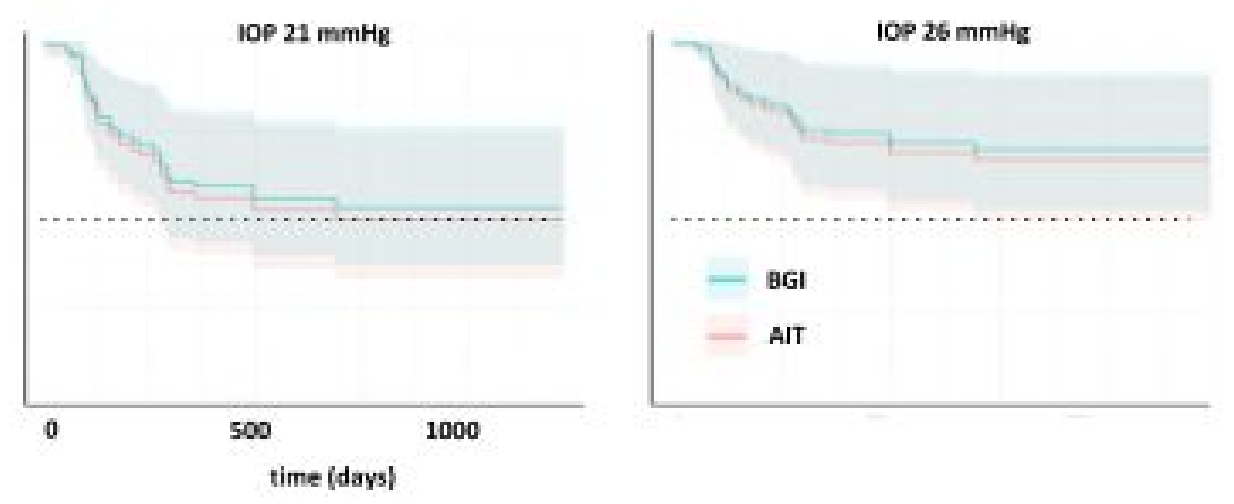
Fig 5. Cox Hazard modelling of BGI and AIT with a target IOP of 16, 21 and $26 \mathrm{mmHg}$. Performance of Baerveldt glaucoma drainage implants (BGI) and ab interno trabeculectomy (AIT) was similar in patients matched preoperatively.

After AIT, the only complication noted was cystoid macular edema (CME) that occurred in a single case which was combined with phacoemulsification. After BGI, complications included hypotony associated with choroidal detachment past three months (two patients), CME (one patient), conjunctival retraction that required surgical revision (one patient), and aqueous misdirection (one patient).

\section{Discussion}

In this study, we found that Trabectome-mediated ab interno trabeculectomy (AIT) and Baerveldt glaucoma drainage devices (BGI) achieved a similar IOP reduction but that BGI had a higher rate of complications compared to AIT. Until recently, AIT had not been directly compared to traditional glaucoma surgeries because the indications and performance were considered too different. Our prior study suggested that AIT and Ahmed glaucoma drainage devices can achieve a comparable pressure reduction when matched carefully on preoperative conditions [5].

AIT enhances the normal, conventional outflow by removing the trabecular meshwork that constitutes the majority of outflow resistance $[17,18]$. In contrast, epibulbar glaucoma drainage implants bypass both the conventional and the uveoscleral route entirely but are subject to encapsidation that is the result of fibrosis and a chronic foreign body reaction [19]. We recently expanded our practice pattern to use AIT as a first surgery for more than mild glaucoma because our studies suggested that AIT can be used after failed trabeculectomy [10], in narrow angles [20] and for more severe glaucoma [6-8]. BGIs lowered IOP to a similar extend as trabeculectomies in a randomized controlled trial but had a higher success rate [14]. In the present study the success rates are relatively similar. 
This study has several limitations, starting with a relatively small number of patients caused by a limited recruitment window from a tertiary referral center. We excluded cases where AIT is contraindicated, notably active neovascular glaucoma. This could bias the results toward AIT by excluding the most complex cases of glaucoma where IOP reduction with AIT (but not BGI) [21] might not be possible. We also excluded cases with previous glaucoma surgery which may help explain the relatively high final IOP in the BGI group. The IOP after BGI only decreased from 21.6 to $15 \mathrm{mmHg}$. Although this is a $31 \%$ decrease, lower IOP's were reached in large prospective trials such as the TVT (3 year IOP of $13.3 \mathrm{mmHg}$, a $47 \%$ decrease) [14], and the pooled Ahmed Baerveldt comparison (3 year IOP of $13.8 \mathrm{mmHg}$, a $57 \%$ decrease) [4].

\section{Conclusions}

Using propensity score matching, AIT resulted in an IOP reduction, final IOP and success rate that was similar to BGI. Patients who underwent AIT required fewer medications and had fewer complications. 


\section{References}

1. Lloyd MA, Baerveldt G, Heuer DK, Minckler DS, Martone JF. Initial clinical experience with the baerveldt implant in complicated glaucomas. Ophthalmology. Elsevier; 1994;101: 640-650.

2. Christakis PG, Kalenak JW, Tsai JC, Zurakowski D, Kammer JA, Harasymowycz PJ, et al. The Ahmed Versus Baerveldt Study: Five-Year Treatment Outcomes. Ophthalmology. 2016;123: 2093-2102.

3. Britt MT, LaBree LD, Lloyd MA, Minckler DS, Heuer DK, Baerveldt G, et al. Randomized clinical trial of the 350-mm2 versus the 500-mm2 Baerveldt implant: longer term results: is bigger better? Ophthalmology. 1999;106: 2312-2318.

4. Christakis PG, Zhang D, Budenz DL, Barton K, Tsai JC, Ahmed IIK, et al. Five Year Pooled Data Analysis of the Ahmed Baerveldt Comparison Study and the Ahmed Versus Baerveldt Study. Am J Ophthalmol. 2017; doi:10.1016/j.ajo.2017.01.003

5. Shazly T, Waxman S, Kola S, Kaplowitz KB, Brown EN, Loewen NA. Similar Performance of Trabectome and Ahmed Glaucoma Devices in a Propensity Score Matched Comparison. ResearchGate preprint. 2017; doi:10.13140/RG.2.2.30723.50727

6. Roy P, Loewen RT, Dang Y, Parikh HA, Bussel II, Loewen NA. Stratification of phaco-trabectome surgery results using a glaucoma severity index. BMJ Ophth in press. 2017; doi:10.13140/RG.2.1.1244.5849

7. Loewen RT, Roy P, Parikh HA, Dang Y, Schuman JS, Loewen NA. Impact of a Glaucoma Severity Index on Results of Trabectome Surgery: Larger Pressure Reduction in More Severe Glaucoma. PLoS One. 2016;11: e0151926.

8. Dang Y, Roy P, Bussel II, Loewen RT, Parikh H, Loewen NA. Combined analysis of trabectome and phaco-trabectome outcomes by glaucoma severity. F1000Res. 2016;5: 762.

9. Mosaed S. Effect of Trabectome in Patients with Prior Failed Tube Shunts Surgery. The Association for Research in Vision and Ophthalmology; 2014. pp. 3178-3178.

10. Bussel II, Kaplowitz K, Schuman JS, Loewen NA, Group TS, Others. Outcomes of ab interno trabeculectomy with the trabectome after failed trabeculectomy. Br J Ophthalmol. BMJ Publishing Group Ltd.; 2014;99: 258-262.

11. Wecker T, Neuburger M, Bryniok L, Bruder K, Luebke J, Anton A, et al. Ab Interno Trabeculectomy With the Trabectome as a Valuable Therapeutic Option for Failed Filtering Blebs. J Glaucoma. 2016;25: 758-762.

12. Sekhon JS. Multivariate and propensity score matching software with automated balance optimization: the matching package for R. papers.ssrn.com; 2011; Available: https://papers.ssrn.com/sol3/papers.cfm?abstract_id=1009044

13. Diamond A, Sekhon JS. Genetic Matching for Estimating Causal Effects: A General Multivariate Matching Method for Achieving Balance in Observational Studies. Rev Econ Stat. 2013;95: 932-945.

14. Gedde SJ, Schiffman JC, Feuer WJ, Herndon LW, Brandt JD, Budenz DL, et al. Treatment outcomes in the Tube Versus Trabeculectomy (TVT) study after five years of follow-up. Am J Ophthalmol. 2012;153: 789-803.e2.

15. Sekhon JS. Multivariate and propensity score matching software with automated balance optimization: the matching package for R. papers.ssrn.com; 2011; Available: https://papers.ssrn.com/sol3/papers.cfm?abstract_id=1009044

16. Core Team R. R: A Language and Environment for Statistical Computing [Internet]. Vienna, Austria: R Foundation 
for Statistical Computing; 2016. Available: http://www.R-project.org/

17. Grant WM. Clinical measurements of aqueous outflow. Am J Ophthalmol. 1951;34: 1603-1605.

18. Toris CB, Koepsell SA, Yablonski ME, Camras CB. Aqueous humor dynamics in ocular hypertensive patients. J Glaucoma. 2002;11: 253-258.

19. Kim YG, Hong S, Lee CS, Kang SY, Seong GJ, Ma KT, et al. Level of vascular endothelial growth factor in aqueous humor and surgical results of ahmed glaucoma valve implantation in patients with neovascular glaucoma. J Glaucoma. journals.Iww.com; 2009;18: 443-447.

20. Bussel II, Kaplowitz K, Schuman JS, Loewen NA, Trabectome Study Group. Outcomes of ab interno trabeculectomy with the trabectome by degree of angle opening. $\mathrm{Br} \mathrm{J} \mathrm{Ophthalmol.} \mathrm{2015;99:} \mathrm{914-919.}$

21. Kolomeyer AM, Seery CW, Emami-Naeimi P, Zarbin MA, Fechtner RD, Bhagat N. Combined pars plana vitrectomy and pars plana Baerveldt tube placement in eyes with neovascular glaucoma. Retina. 2015;35: 17-28. 